\title{
Table of legislation, treaties, conventions and other international
} \section{instruments}

Agreement on Common Effective

Preferential Tariff of the

ASEAN Free Trade Area

(CEPT-AFTA)

2,45

ASEAN Agreement on the

Movement of Natural Persons

(MNP Agreement) 2012 ....... 136

ASEAN Agreement on the

Promotion and Protection of

Investments (ASEAN IGA)

1987........ 7, 47, 48, 49, 51, 67, 156

Amending Protocol 1996........... 7, 47

ASEAN Australia-New Zealand

Free Trade Area (AANZFTA)

2010 41, 48, 134, 181-189, 191, 193-196, 197, 199

Ch 8 183

Ch 9 183,195

Ch 11 $182,183,184,186$

Art 2(5) 197

Art 3(2) 184

Art 4 184

Arts 5-11 183

Art 6 184,185

Art 6(2)(b) 184

Art 7 184, 197

Art 8. 185

Art 8(3) 185

Art 9 185

Art 12 184,186

Arts 13-14 183

Art 13(1) 185

Art 13(2) 186

Art 13(4) 186

Art 13(5) 186

Art 13(6) 186 Art 13(8)
Art 13(9) ...................................186

Art 13(10-12) .............................186

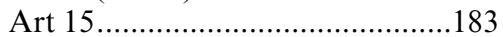

Art 16............................. 184, 185,

186

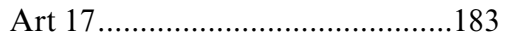

Arts 18-28 ...................................183

Art 23(3) .....................................197

ASEAN Charter 2007 ................2, 4, 6

Ch 1 ............................................. 4

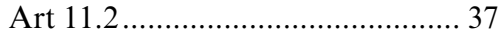

Art 14.5 _...................................... 37

Art 27........................................ 38

Art 29....................................... 37

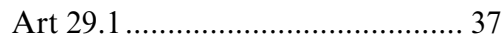

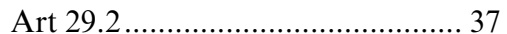

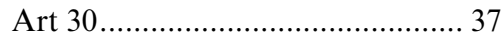

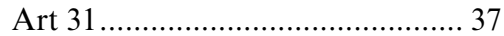

Art 32 ......................................... 37

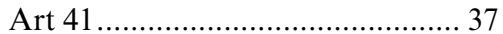

Annex 1.................................. 4,37

Annex 2................................. 4,37

Annex 3 ........................................ 4

Annex 4........................................ 4

ASEAN China Free Trade Area

(ACFTA) 2009... 48, 182, 189-190, 193-196, 197, 198,

199

Art 1..........................................189

Art 4.........................................190

Art 5.......................................189

Art 5(3) .....................................199

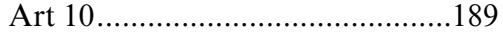

Art 23 ...........................................199

ASEAN-China Investment

Agreement into force on 1

January 2010 199 
ASEAN Comprehensive Investment Agreement (ACIA) ...... 7, 8, 9, 14, $15,22,25,26,28,36-38,45$, $46,47,48,49,50,51,65-158$, $162,163,181,193-196,197$, 198, 199, 203, 204, Annex 1,

Annex 2

Art 3 49,72

Art 3.2 ... 73

Art 4 $77,78,79$ Art 4(d) 80,81

Art 5 85

Art 6 . $90,91,93,94$,

Art 7. 103, 107

Art 8 . .103

Art 9. .. 70

Art 10 111

Art 11 117,123

Art 12 128

Art 13 129

Art 13(4) 132

Art 14(5)

Art 15 128

Art 16 .134

Art 17 132,153

Art 18 $141,142,143$

Art 19 142,153

Art 21 154-158

Art 21(2) 138

Art 22 138

Art 24 .135

Art 25

Art 29 72

Art 30 164

Art 31 164,165

Art 32. 164,166

Art 33 ........ 164,166

Art 34. 164,167

Art 35 . 167,168

Art 36.5 167,168

Art 41 .169

Annex 172

Annex 2 79, 107

Protocol to Amend ACIA ............204 ASEAN Declaration 1967

(Bangkok Declaration) 2 ASEAN Economic Community 2015 (AEC) $26,45,46$
ASEAN Framework Agreement on Trade in Services (AFAS) 1995. $2,45,46,50$

ASEAN Investment Area

Framework Agreement (AIA) 1998... 7, 36, 47, 48, 49, 51, 68, 209

Art 8(3) 93,94

Amending Protocol .................. 7, 47

ASEAN-Korea Free Trade Area

(AKFTA) 2009 ................48, 182, 190-191, 193-196

ASEAN-Korea Investment Agreement (AKIA) 191, 197, 198, 199 Art 4(3)(b) .................................198 ASEAN-PRC FTA 2005 .................. 38

ASEAN Protocol on Enhanced Dispute Settlement Mechanism $2004 \ldots \ldots \ldots \ldots \ldots \ldots \ldots \ldots . . . . . . .38,101,173$

ASEAN Trade in Goods Agreement (ATIGA) 2009 ......... 2, $36,45,46$ Annex 2...................................... 46

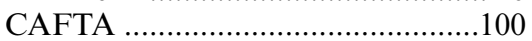

Art 10.4:1 .................................... 96

Cebu Declaration on Acceleration of the Establishment of an ASEAN Community ... 4, 5, 15, 45

Convention on the Recognition and Enforcement of Foreign Arbitral Awards 1958 (New York Convention) 101,102 , $169,170,173$

Art 1 172

Framework Convention on Tobacco Control (FCTC) 154

General Agreement on Trade in Services (GATS), WTO ......46, 68, 104,129

Art I:2 …....................................... 46

Art XIX …..................................... 68

GATT 1947.....................108, 144, 146

GATT 1994

Art III ................ 104, 107, 111, 148

Art III:1 ......................................107

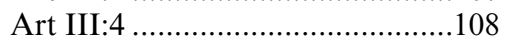

Art III: 8 ....................................107

Art IV .........................................111

Art IX .........................................104

Art XI ...........107, 108, 109, 110, 143 
Art XI:1 109,110

Art XIII: 1 109

Art XVI 107

Art XIX 107

Art XX. $107,142,143,144,145$, $146,147,148$

Art XX(b) $144,145,146,147$,

$\operatorname{Art} \mathrm{XX}(\mathrm{g})$ $144,145,146$, 147

Art XXI 107

ICSID Additional Facility Rules....167, 172

ICSID Convention... 101, 151, 167, 190

ICSID Rules 169,172

IMF Articles of Agreement

Art VIII, s 2(a)

Kuala Lumpur Declaration on the

Establishment of the ASEAN

Charter

Kuala Lumpur Declaration on a

People-Oriented, People-

Centred ASEAN on 27 April 2015 132

Kuala Lumpur Declaration on ASEAN 2025.

Malaysia-Australia Free Trade Agreement (MAFTA) signed 2012, into force 2013........ 187, 188 NAFTA $13,40,41,85,97$, 118

Ch 11 40,105

Art 1102 88,105

Art 1102(1) 87

Art 1102(2) 86,87

Art 1103 ..105

Art 1105 $98,105,118$

Art 1106 104,105

Art 1107 .105

Art 1108

97, 104

Art 1108(1) .105

Art 1108(3) 105

Art 1108(7) 105

Art 1110 105

Annex II 105

Regional Comprehensive Economic Partnership (RCEP)...... 36, 38, 39

Singapore-Australia Free Trade Agreement (SAFTA) 2003, as amended $186-187$ 4
Thailand - Australia Free Trade Agreement (TAFTA) 2005.

Trade-Related Aspects of Intellectual Property Rights (TRIPS) Agreement

Trade and Investment Measures (TRIMs) Agreement, WTO .... 52, 79, 104, 106, 107, 108, 110,111

Art 4.1 107

Annex. 108,109

Trans-Atlantic Trade and Investment Partnership (TATP) 40

Treaty of Amity and Cooperation (TAC) 1976 (Bali Concord I) ...... 2

Treaty of Rome 1957........................ 1

Treaty of Amity and Economic Relations (AER) 1966.............102,

TPP 2016 103

UN Charter 39-41

United Nations Conference on Trade and Development (UNCTAD) (2004)

Transparency, Geneva 137

United Nations Conference on Trade and Development, Taking of Property, 12, UN Doc. UNCTAD/ITE/IIT/15 (2000) (UNCTAD) 125

UNCITRAL Arbitration Rules .....161, $162,167,169$

Vienna Convention on the Law of Treaties (VCLT) ........................147

Art 26.

\section{BITs, FTAs etc}

Argentina-Italy BIT........................101

Argentina-Spain BIT 1991 ............... 98

Canadian Model BIT 2003

Art 1 ..... 76

Canadian Model FIPA of 2003. 76

Canadian Model FIPA of 2004

Art 3.1 85

Chile-Spain BIT ............................... 98

China-Korea BIT ...........................120

Czech-Israel BIT ............................157 
France Model BIT 2006

$$
\text { Art } 4 \text {. }
$$

Hong Kong, China-Australia BIT..154,

India Model BIT 2003

$$
\text { Art } 4 \text {. }
$$

93,94

Japan-ASEAN EPA 2008................. 38

Japan-Korea BIT 2002 .................... 73

Art 23.1

.73

Jordan-Singapore BIT, 2004.

Malaysia-New Zealand FTA (MNZ FTA) 187,188

NZ-Singapore CEP 124

NZ-China FTA (2008) 124

PRC-Japan-Republic of Korea Trilateral Investment Treaty..... 36, $38-39$

Pakistan-Switzerland BIT 155

Singapore-Jordan BIT, concluded 2004, into force 2005. 142

Singapore-Jordan Free Trade Agreement 2005

Singapore-New Zealand FTA (SNZ) 187,189

Singapore-United States FTA 2003

Art 15.13(2) .138

Art 21.4

Switzerland-Pakistan IIA

Thailand-Japan PTA

120

Thailand-New Zealand FTA (TNZ) 187,189

UK-Belize BIT

Article 3.1 96

US Model BIT 2004

US Model BIT 2012

85

USA-Australia FTA

US-Ecuador BIT.

US-Honduras BIT

Article II.1. 76,77

USA-Uruguay BIT .96

UK-Vanuatu BIT 2003

Arts 1-11

120

Art 3.3 .. 99

Art 9

99

United States-Thailand TIFA 2002

\section{National Legislation}

\section{Australia}

Tobacco Plain Packaging Act 2011

\section{Brunei}

Strata Title Act 2009 58

\section{Cambodia}

Law of Investments 1994 ................. 54

Law on Expropriation......................... 58

\section{Indonesia}

Investment Law No 5/2007 .............. 54

Presidential Decree 39/2014 ............. 54

\section{Lao PDR}

Law on Investment Promotion 2010

\section{Malaysia}

Companies Act 1965 62

Investment Development Authority Act

Industrial Coordination Act. 55

\section{Myanmar}

Arbitration Law 55,173

Companies Law 55

Foreign Investment Law.............59, 62

Investment Law. 55,59

\section{Philippines}

Banking Law 2014 ........................... 60

Foreign Investment Act 1991 ............. 60

Special Economic Zone Act 1995 ..... 60

\section{Thailand}

Foreign Business Act (FBA) 1999 ... 55,

Alien Employment Act B.E. 2521 (1978) ......................................... 56

Investment Promotion Act B.E. 2520 (1977) 56

\section{Vietnam}

Investment Law 2014 $56,60,63$ 